\title{
Erratum: Otto refrigerator based on a superconducting qubit: Classical and quantum performance [Phys. Rev. B 94, 184503 (2016)]
}

B. Karimi and J. P. Pekola

(Received 9 January 2017; published 20 January 2017)

DOI: 10.1103/PhysRevB.95.019903

In the text the following corrections are to be applied: Just after Eq. (3), $\left|\left\{R_{j}\left[1+Q_{j}^{2}\left(\frac{\omega}{\omega_{L C, j}}-\frac{\omega_{L C, j}}{\omega}\right)^{2}\right]\right\}\right|^{-2}$ should be replaced by $\left\{R_{j}^{2}\left[1+Q_{j}^{2}\left(\frac{\omega}{\omega_{L C, j}}-\frac{\omega_{L C, j}}{\omega}\right)^{2}\right]\right\}^{-1}$, and accordingly in the appendix Eq. (A2), $\left|\operatorname{Re}\left[Y_{j}(\omega)\right]\right|^{2}$ should read as $R_{j}^{-1} \operatorname{Re}\left[Y_{j}(\omega)\right]$ and $\left|R_{j}+i\left[\omega L_{j}-1 /\left(\omega C_{j}\right)\right]\right|^{-2}$ as $\left\{R_{j}^{2}\left[1+Q_{j}^{2}\left(\frac{\omega}{\omega_{L C, j}}-\frac{\omega_{L C, j}}{\omega}\right)^{2}\right]\right\}^{-1}$. In Eq. (10), $\left[1+Q_{k}^{2}\left(\frac{\omega}{\omega_{L C, k}}-\frac{\omega_{L C, k}}{\omega}\right)^{2}\right]^{2}$ is to be replaced by $\left[1+Q_{k}^{2}\left(\frac{\omega}{\omega_{L C, k}}-\frac{\omega_{L C, k}}{\omega}\right)^{2}\right], k=1,2$.

These typographical errors appear only in the text. The figures and conclusions in the original paper are based on the correct expressions. 